\title{
Téoros
}

Revue de recherche en tourisme

\section{L’archipel après Archipel...}

\section{Jean Décarie}

Volume 6, numéro 2, juillet 1987

\section{Le Saint-Laurent magnétique}

URI : https://id.erudit.org/iderudit/1080511ar

DOI : https://doi.org/10.7202/1080511ar

Aller au sommaire du numéro

\section{Éditeur(s)}

Université du Québec à Montréal

\section{ISSN}

0712-8657 (imprimé)

1923-2705 (numérique)

Découvrir la revue

Citer cet article

Décarie, J. (1987). L’archipel après Archipel... Téoros, 6(2), 33-34.

https://doi.org/10.7202/1080511ar d'utilisation que vous pouvez consulter en ligne.

https://apropos.erudit.org/fr/usagers/politique-dutilisation/ 


\section{L'archipel après Archipel...}

par Jean Décarie*

Le 31 mars dernier, le Parc national Archipel prenait en catimini le chemin des tablettes gouvernementales où il allait rejoindre le projet Archipel dont il était le rejeton. L'expérience se terminait ainsi 8 ans, mois pour mois - et 55 millions de dollars - après son lancement en ayril 1979.

Le projet Archipel s'était defini, des le départ, dans l'etude de pré-faisabilité qui en avait ćtabli les buts et les objectifs, les principes et les moyens, comme ce que l'on appelle un "plan d'aménagement hydraulique a fins multiples" , c'est-à-dire un plan d'aménagement du territoire, d'un bassin hydrographique, à partir d'un controble et d'un partage de la ressource hydrique entre ses différents usagers. De tels "plans" sont fort connus, depuis la plus haute antiquité, dans les pays subtropicaux où ils ont été à l'origine du droit et de l'urbanisme. Plus près de nous, citons l'exemple de la Tennessee Valley, de la vallée du Rhône, ou même du plan de gestion du Saint-Laurent et des Grands Lacs.

\section{Développer les fonctions sociales de l'eau}

Gräce aux caractéristiques hydrographiques exceptionnelles de l'archipel de Montréal qui forme tout à la fois un systeme hydraulique et un écosystème hydrique en équilibre, le projet Archipel aurait été le premier du genre à s'appliquer en milieu urbain d'abord et, en conséquence, au développement des fonctions sociales de l'eau, de façon à rétablir l'équilibre avec les fonctions économiques (transport, énergie, dilution). Rappelons que celles-ci avaient prealablement exploite le site et produit la ville, la coupant cependant de la ressource pour la satisfaction de ses besoins sociaux récréatifs secondaires, devenus prioritaires et majoritaires avec le développement urbain.

Le projet Archipel était essentiellement un projet d'urbanisme visant à réintégrer l'eau comme habitat urbain - et non seulement faunique - dans le vécu et le tissu urbain, comme élément déterminant du cadre et de

\footnotetext{
- Jean Décarie est consultant en emvironnement ot il
} fut un des concepteurs du Parc national Archipel. la qualite de vie urbaine des trois millions de Montréalais. Le moyen technique était la gestion ou la régulation hydraulique qui devait permettre un meilleur contróle des variations de niveaux et de la repartition des débits entre les plans d'eau, les saisons et les usages; de mème, le moyen economique était-il - au départ tout au moins - une usine hydroélectrique placée dans un canal d'évacuation de crue contournant les rapides de Lachine; mais la finalité a toujours été urbaine et sociale. Le projet Archipel était le premiér projet d'aménagement véritablement écologique au Québec.

Les difficultés institutionnelles, plus qu'économiques ou environnementales, de l'etude de faisabilité qui devait porter essentiellement sur ces moyens, donc sur le couple bydraulique-hydroelectrique, ont amené à anticiper les résultats, à savoir le volet "aménagement des rives" dont plus de $80 \%$ des élements relevaient du secteur conservation-récréation. Ce "'devancement" devait permettre au ministere du Loisir, de la Chasse et de la Péche du Québec (MLCP), responsable de ce secteur, de recuperer non seulement ce volet mais la finalité même du projêt. II devait de même permettre au gouvernement, par une pirouette pré-électorale, de laisser HydroQuebec couler définitivement le projet Archipel et de lui substituer l'annonce positive du Parc national Archipel.

Le Parc national Archipel était un hybride entre, d'une part, le contenu des programmes d'équipement et d'aménagement par réseau thématique (faune, nautisme, baignade, etc.) elaboré par le MLCP dans le cadre de sa participation à l'étude de faisabilité du projet Archipel et, d'autre part, le contenant du projet d'une politique des parcs urbains pour Montréal élaboré en parallèle par le même ministère pour pallier au déficit d'espaces verts et ouverts de la région métropolitainé, tant au niveau local que régional.

A cette échelle, le projet de politique proposait $\mathrm{d}^{+} u n e$ part un réseau vert linéaire, véritable système de communication-loisir, empruntant littéralement les friches industrielles et les emprises du réseau de transport et du réseau communautaire, traversant les quartiers et reliant le centre à la périphérie et aux plans d'eau de l'Archipel, derniers grands espaces à la fois libres et naturels de la région; il proposait, d'autre part, de réunir ceux-ci en une ceinture bleue, pour lesquels une structure de gestion et de développement associative était suggérée, chacun des six plans d'eau faisant l'objet d'un parc régional autonome, optimisant et proposant ses caractéristiques spécifiques à la clientèle métropolitaine dans une perspective d'appropriation, de différentiation et d'émulation.

\section{Une perspective touristique et économique}

Cette perspective était nettement touristique et économique. Les parcs et réseaux verts et bleus s'adressaient en effet moins aux besoins sociaux locaux qu'ta la population régionale attirúte par la mise en valeur des ressources territoriales et patrimoniales de l'archipel. A l'attrait du tourisme interne $s^{4}$ ajoutait celui, possible, du tourisme externe à la région de Montréal par la mise en valeur non plus seulement des divers éléments de l'archipel mais, à travers eux, de l'archipel tout entier, comme phénomène naturel exceptionnel en soi, rendu plus exceptionnel encore par son occupation et sa mise en valeur urbaine que le projet visait précisément à parfaire.

L'archipel de Montréal, comme système hydrographique, représente en effet, davantage que le Mont-Royal, l'élément naturel le plus déterminant de l'environnement et de la geographie urbaine de Montréal, à la fois enveloppe et énergie; il la distingue en outre parmi les grandes métropoles occidentales considérées comme destinations touristiques concurrentes.

A cet égard, au contraire du Québec dont le produit touristique associe le culturel au naturel, l'image de Montréal est essentiellement basée sur le seul différentiel culturel français. Il est indispensable d'asseoir et d'assurer cette image dans un décor naturel, de lui donner un espace et un paysage qui pourrait devenir, éventuellement, un attrait en soi. La Seine n'est pas le SaintLaurent ni même 1'Outaouais et pourtant elle participe autant que la tour Eiffel de 
l'image touristique parisienne et mème francaise... Les rapides de Lachine ne sont pas le Niagara, mais Niagara Falls n'est pas Montréal! Et allez donc sauter les chutes en pneumatique!

La plupart des grandes villes sont situées sur un bord de mer ou de lac, ou sur un fleuve. Mais aucune n'est assise, comme Montréal, à cheval sur un ressaut hydraulique de 50 metres où se percutent et se précipitent un fleuve et une rivière aussi importants en quantité (8500 cubes/seconde) et en qualité (deux écosystèmes continentaux différents), déployant trois lacs et trois chenaux, 21 rapides et 325 iles, 1000 kilomètres de rives, le tout autour et au coeur d'une agglomération de trois millions d"habitants!

Le problème est là, justement: cettè ville tourne le dos aux eaux qui l'ont produite! Elle n'est pas aménagée sur l'eau, mais contre l'eau, difficilement accessible et de toute façon fortement polluéc. Autrement dit - et c'est là un des principes fondamentaux du tourisme -, comment intéresser le tourisme à une ressource qui n'intéresse pas d'abord les Montréalais, qu'ils n'ont pas aménagé a leur propre image et qui ne constitue pas un paysage humain, un produit touristique intégré?

\section{Réhabiliter et réapproprier les plans d'eau}

L'élaboration de ce produit doit passer par une réhabilitation et une réappropriation de ses plans d'eau, comme espaces urbains, par la ville. Cela veut dire d'une part, physiquement, retourner la ville sur l'eau, revenir habiter au bord de l'eau, créer un véritable front de mer urbain. A cet égard, les tours $d$ "habitations qui poussent depuis quelques années en rive - résultats indirects du projet Archipel = ne sont pas à condamner; au contraire, elles expriment la valeur de localisation marginale de l'habitation en rive, qui est une valeur de loisir; elles récupèrent la plus-value de l'investissement dans ce début d'optimisation et d'exploitation des plans d'eau; elles permettent à cent personnes - et non à quelques-unes de jouir de la méme superficie en rive; les droits de vue et de passage en rive y sont plus facilement contrôlables, négociables, par les municipalites. De méme, la concentration des densités sur certains segments plus favorables de rives permet de dégager d'autres sites, a haut potentiel faunique ou récréatif, pour les parcs publics dont la fréquentation et l'animation n'en seront que plus actives. L'imposition d'un zonage vert exclusif sur toutes les rives serait antinaturelle autant qu'anti-sociale.

D'autre part, cela veut également dire investir d'abord en imagination autant et sinon plus qu'en millions dans les plans d'eau eux-memes. Le timide mouvement actuel risque en effet de s'arrêter rapide-
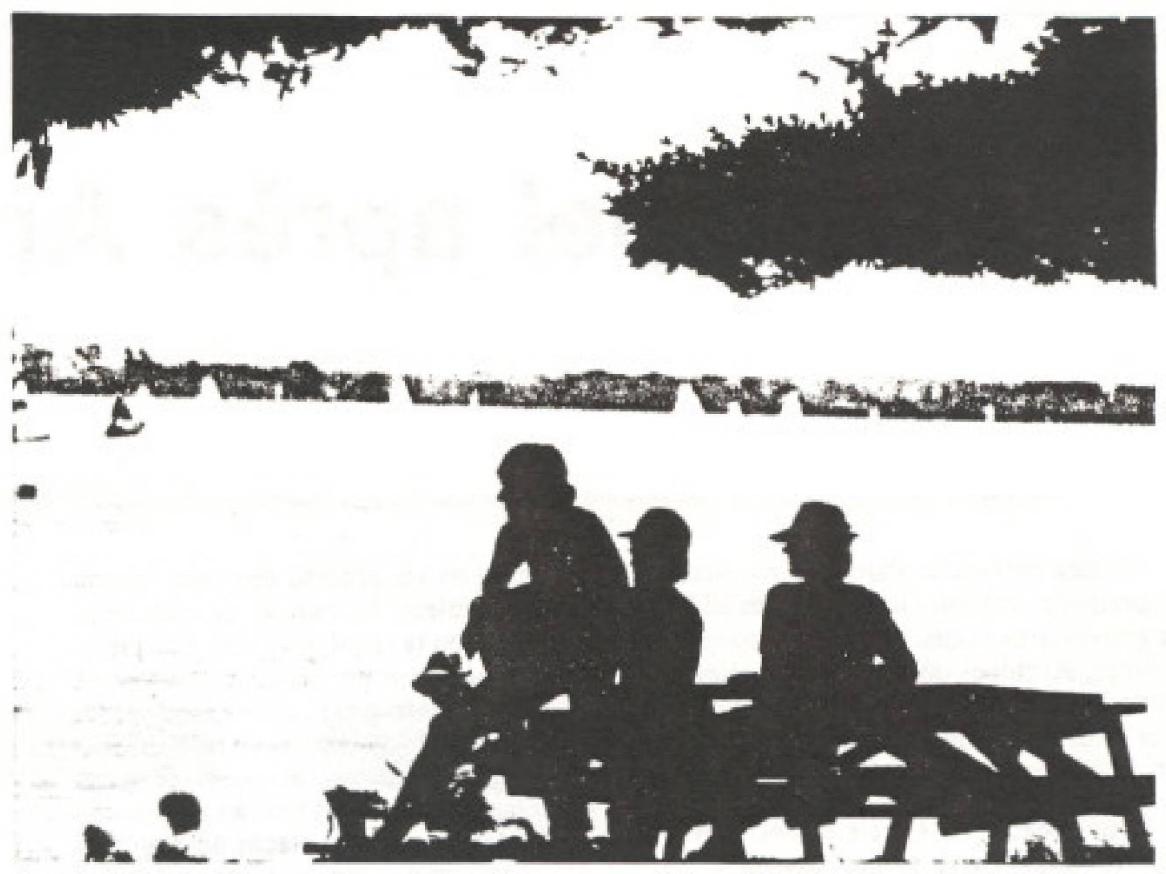

Parc Paul-Sauve.

ment si rien n'est fait pour poursuivre l'assainissement et l'aménagement des eaux. Le projet Archipel ou plutôt le Parc national Archipel reste entièrement à revoir a cet effet, mais dans une nouvelle perspective politique et institutionnelle, cellelà même tracée par le projet politique dès 1983 où, au lieu d'un gros parc national cachant un petit programme d'aide unilatérale aux municipalités sous couvert d'un discours politique, on reviendrait a un système d'action sophistiqué et différencié, associant tous les paliers de gouvernement à I'entreprise privée, commerciale comme communautaire, à l'intérieur de diverses formules de gestion mixte, selon la nature et l'échelle des éléments et des projets.

Une certaine "remunicipalisation" du Parc national Archipel est déjà commencée avec la prise en mains de la partie centrale du "réseau vert intermédiaire" par la nouvelle administration de Montréal et par son ouverture à la négociation avec les municipalités périphériques pour la mise en valeur des espaces-ressources situés pour la plupart en dehors des limites de la ville de Montréal; citons, par exemple, l'aménagement, avec le fédéral, d'un parc-plage de 15 kilomètres de long sur la rive extérieure de la digue de la Voie maritime dans le bassin de La Prairie, seul endroit de l'archipel où l'eau est naturellement baignable, accessible de surcroit par métro depuis l'ille Notre-Dame ou l'estacade du pont Champlain!

Citons également l'initiative de la ville de LaSalle de proposer la création d'un parc national ou international et interrives aux rapides de Lachine, encadrant leur inscription sur la liste du patrimoine mondial de I'Unesco! Rappelons de mëme les projets de Lachine et de Longueuil - qui donneraient ainsi l'exemple au Vieux-Montréal - de revivifier leurs vieux centres-villes en les rebranchant sur l'eau, sous forme de ports de mer urbains à haute intensité sociale et commerciale: ce ne seront plus des marinas exclusives mais des équipements urbains, des secteurs fonctionnels nouveaux, comme on en trouve dans toutes les grandes villes du littoral américain.

Plusieurs autres projets ont été identifiés et soulevés lors des etudes et des tables de concertation par bassin du Parc National Archipel - et que le parc national lui-mêmé, par sa définition et sa gestion arbitraire et autoritaire a empêché. Ce n'est pas d'un parc national que l'archipel a besoin - seuls les rapides de Lachine répondent de toute façon à la définition de parc national - pour son développement touristique et récréatif, mais d'un processus permanent de planification concertante reposant d'abord sur les producteurs d'offres de services, ensuite sur les consommateurs communautaires, enfin seulement sur l'aide supplétive et incitative de l'etat, au niveau de ses propres responsabilités bien sür - I'aménagement et la gestion de la faune par exemple - mais aussi et peut-être surtout de ses propriétés. le domaine privé de l'État, qui représente la seule grande réserve d'espaces libres de Montréal et qui devrait être ouvert au public sous gestion associative.

Le tourisme, interne ou externe, pourra alors peut-être ajouter autour de la montagne - musée, unjversité, oratoire et une vue plongeante sur tout le reste - un tour de l'île 20 fois plus long et lui donnant non plus une vue superficielle de l'ensemble, mais une expérience amphibie, symbiotique, avec tout l'écosystème urbain de Montréal. $f$ 